\title{
Analysis of the Therapeutic Effects of Staged Posterior-Anterior Combined Surgery for Cervicothoracic Segmental Tuberculosis with Kyphosis in Pediatric Patients
}

\section{Haopeng Luan \\ Qiang Deng (D) \\ Weibin Sheng \\ Mardan Mamat \\ Hailong Guo \\ Huaqiang $\mathrm{Li}$}

Department of Spine Surgery, Xinjiang Medical University Affiliated First Hosptial, Urumqi, Xinjiang, People's Republic of China
Correspondence: Qiang Deng Department of Spine Surgery, Xinjiang Medical University Affiliated First Hospital, Urumqi, Xinjiang, People's Republic of China

Tel +86 13325636562

Email dengqiang_abc@163.com
Objective: The present study aimed to investigate the surgical efficacy of staged posterioranterior combined surgery for the treatment of cervicothoracic segmental tuberculosis (TB) with kyphosis in pediatric patients.

Methods: The clinical data of 15 pediatric patients admitted to our hospital from January 2010 to December 2017 who underwent staged posterior-anterior combined surgery for cervicothoracic segmental TB with kyphosis were collected. A posterior median incision was made for patients after general anesthesia. Autologous bone particles or allogeneic bone particles were taken, trimmed, and placed in the articular eminence of the diseased vertebral body. Fifteen pediatric patients underwent second-stage lesion removal using the anterior approach. The left sternocleidomastoid muscle was selected as the medial oblique incision approach. The abscess and caseous necrotic material were removed and sent for pathological examination. The sagittal and coronal parameters (including the local Cobb angle, the sagittal vertical axis [SVA], and the coronal balance distance [CBD]) were measured at three time points: preoperatively, postoperatively, and at the final follow-up. The American Spinal Injury Association's spinal-cord injury classification, the Japanese Orthopaedic Association's (JOA) cervical spine function score, the neck disability index (NDI), and the visual analogue score (VAS) for cervicothoracic segment pain were adopted for the assessment of functional improvement and quality of life.

Results: All 15 pediatric patients completed the surgery successfully, with an operation duration of $3.56 \pm 0.68 \mathrm{~h}$, an intraoperative hemorrhage of $289.7 \pm 84.3 \mathrm{~mL}$, an average fixation of $7.3 \pm 1.8$ segments, and a follow-up duration of $28.1 \pm 9.7$ months. The preoperative and postoperative sagittal local Cobb angle was $67.06 \pm 17.54^{\circ}$ vs $19.48 \pm$ $2.32^{\circ}(\mathrm{P}<0.01)$, the SVA was $35.19 \pm 10.69 \mathrm{~mm}$ vs $7.67 \pm 1.40 \mathrm{~mm}(\mathrm{P}<0.01)$, and CBD was $22.58 \pm 7.59 \mathrm{~mm}$ vs $8.99 \pm 1.25 \mathrm{~mm}(\mathrm{P}<0.01)$. The levels of the postoperative erythrocyte sedimentation rate and C-reactive protein were significantly lower in all patients. The preoperative and postoperative JOA scores were $8.93 \pm 3.51$ and $14.67 \pm 1.34$, respectively, the preoperative and postoperative VAS was $7.40 \pm 1.35$ and $2.67 \pm 0.62$, respectively, and the preoperative and postoperative NDI was $32.67 \pm 4.83$ and $13.73 \pm 2.08$, respectively. There were statistically significant differences in the above indicators before and after surgery $(\mathrm{P}<0.05)$.

Conclusion: In the surgical treatment of cervicothoracic TB with kyphosis in pediatric patients, staged posterior-anterior combined surgery significantly corrects deformity, achieves the safe and effective neurological decompression of the spinal cord, and obtains 
good neurological recovery and bone-graft fusion according to the extent of the involved segments of kyphosis, the characteristics of the lesion, and the degree of neurospinal injury.

Keywords: spinal tuberculosis, cervicothoracic segment, posterior and anterior combined surgery, pediatric patient, therapeutic effect

\section{Introduction}

Spinal tuberculosis (TB) is a common cause of severe kyphosis in many parts of the world. ${ }^{1}$ Due to the unique anatomy of the spine in children, kyphosis caused by TB is more dangerous. ${ }^{2}$ In children, spinal TB progresses slowly or even asymptomatically, while the symptoms of pain, neurological abnormalities, and even deformities are already apparent in children at the time of their visit to hospital. ${ }^{2,3}$ In addition, the cervicothoracic segment is the junction between the physiological cervical lordosis and the thoracic kyphosis and has concentrated stress. Moreover, the diameter of the thoracic spinal canal is small, thus it is difficult to compensate for the blood supply to the upper thoracic medulla, and the symptoms are more serious when kyphosis occurs in this area., Therefore, the treatment of cervicothoracic TB with kyphosis in pediatric patients has become a great challenge for orthopedic surgeons. Although anti-TB drugs are effective in controlling the infection, studies have reported an average incidence of deformity of $15 \%$ in pediatric patients with spinal TB treated with chemotherapy alone. $^{6}$ As a result, Issack et al ${ }^{7,8}$ considered that surgery should be performed in pediatric patients with severe pain and significant kyphosis resulting in neurological impairment. Available methods in the treatment of cervicothoracic TB include conventional anterior cervical approach, anterior approach combined with sternotomy or manubriotomy, standard transclavicular approach, approach by resection of the manubrio-clavicular complex, anterolateral transthoracic approach, and combined approaches. ${ }^{9}$ The aim of the surgery is to completely remove the lesion, correct the kyphosis, prevent the progression of the deformity, and restore the nerve injury. ${ }^{8,10}$ Due to the special and complex anatomical structure of the cervicothoracic segment and considering that children are in the growth and developmental stage, surgical treatment is more difficult. ${ }^{11}$ However, there is still no consensus on the surgical treatment of cervicothoracic segmental TB in pediatric patients, and there are few reports on the staged posterior-anterior approach for the treatment of cervicothoracic segmental TB with kyphosis in pediatric patients. ${ }^{12,13}$ The present study aimed to address this gap by discussing the therapeutic effects and practicalities of this approach.

\section{Materials and Methods}

\section{The Inclusion Criteria}

The inclusion criteria were as follows: (1) patients aged $<14$ years; (2) patients with imaging showing abscesses anterior to the vertebral body and in the spinal canal; (3) patients with combined multi-segmental ( $\geq 3$ segments) cervicothoracic kyphosis; (4) patients with a combination of spinal-cord compression and varying degrees of neurological impairment. The exclusion criteria were as follows: (1) patients with non-TBrelated infectious diseases; (2) patients with congenital spinal deformities and a history of previous spinal surgery; (3) patients not able to tolerate the surgical treatment.

\section{Clinical Data}

A total of 15 pediatric patients with cervicothoracic segmental TB and kyphosis who had undergone staged combined posterior-anterior surgery in the Department of Spine Surgery from January 2010 to December 2017 were included in the present study based on the above inclusion and exclusion criteria. Of these patients, six were male and nine were female, with an average age of 6.8 years (3-12 years). The TB lesions involved four vertebrae in ten cases, five vertebrae in three cases, eight vertebrae in one case, and ten vertebrae in one case. The detailed general characteristics of the patients are illustrated in Table 1.

The clinical manifestations in these patients included systemic symptoms of TB toxicity, such as night sweats, a low fever, loss of appetite, and fatigue. The most common complaints in pediatric patients with early spinal TB are varying degrees of neck stiffness and pain, decreased muscle strength and sensation in the upper extremities, or limited spinal motion. As the disease progresses, it causes compression of the spinal cord, resulting in abnormal posture and even varying degrees of limb paralysis. In the present study, computed tomography (CT) and X-rays showed significant spinal-bone destruction and kyphosis. Magnetic resonance imaging (MRI) showed destruction of the vertebral body and disc, abscess formation, and spinal- 
Table I The General Characteristics and Follow-Up Data in 15 Cases with Cervicothoracic Tuberculosis and Kyphosis

\begin{tabular}{|c|c|c|c|c|c|c|c|c|c|}
\hline \multirow[t]{2}{*}{ Cases } & \multirow[t]{2}{*}{$\begin{array}{l}\text { Agel } \\
\text { Gender }\end{array}$} & \multirow[t]{2}{*}{$\begin{array}{l}\text { Follow- } \\
\text { Up Time } \\
\text { (Months) }\end{array}$} & \multicolumn{3}{|c|}{ ASIA Grade } & \multicolumn{2}{|c|}{$\begin{array}{c}\text { Erythrocyte } \\
\text { Sedimentation Rate } \\
(\mathbf{m m} / \mathbf{h})\end{array}$} & \multicolumn{2}{|c|}{ C-Reactive Protein(mg/L) } \\
\hline & & & Pre-Operative & Postoperative & $\begin{array}{l}\text { Final Follow- } \\
\text { Up Visit }\end{array}$ & $\begin{array}{c}\text { Pre- } \\
\text { Operative }\end{array}$ & $\begin{array}{c}3 \text { Months } \\
\text { Postoperative }\end{array}$ & Pre-Operative & $\begin{array}{c}3 \text { Months } \\
\text { Postoperative }\end{array}$ \\
\hline 1 & 3/Female & 23 & C & $E$ & E & 45 & 4 & 22.88 & 6.37 \\
\hline 2 & I2/Male & 34 & C & C & C & 43 & 3 & 13.58 & 4.69 \\
\hline 3 & 8/Male & 35 & B & D & D & 53 & 11 & 24.56 & 9.32 \\
\hline 4 & I2Female & 29 & C & D & D & 46 & 6 & 14.09 & 7.78 \\
\hline 5 & 6/Male & 40 & C & E & E & 40 & 18 & 47.70 & 11.38 \\
\hline 6 & 2/Male & 29 & D & D & $\mathrm{E}$ & 32 & 4 & 10.10 & 7.65 \\
\hline 7 & 8/Female & 39 & B & C & D & 28 & 3 & 11.05 & 6.79 \\
\hline 8 & 3/Female & 44 & D & E & $\mathrm{E}$ & 18 & 7 & 22.96 & 7.70 \\
\hline 9 & I2Female & 25 & B & D & $\mathrm{E}$ & 62 & 4 & 22.50 & 10.32 \\
\hline 10 & 4/Male & 15 & C & D & $\mathrm{E}$ & 60 & 23 & 9.25 & 7.31 \\
\hline II & 4/Female & 24 & B & C & D & 30 & 3 & 17.40 & 10.13 \\
\hline 12 & 9/Female & 14 & D & E & $\mathrm{E}$ & 26 & 8 & 65.90 & 15.76 \\
\hline 13 & 4/Male & 12 & C & D & E & 34 & 6 & 27.45 & 9.08 \\
\hline 14 & 9/Female & 26 & D & $\mathrm{E}$ & $\mathrm{E}$ & 43 & 16 & 27.36 & 9.44 \\
\hline 15 & 6/Female & 33 & D & E & $\mathrm{E}$ & 42 & 9 & 23.35 & 8.67 \\
\hline
\end{tabular}

nerve compression (Figures 1 and 2). Laboratory test results included an elevated erythrocyte sedimentation rate (ESR), increased levels of C-reactive protein (CRP), and positive cultures for Mycobacterium TB. All pediatric patients completed two weeks of regular quadruple antiTB medication before surgery. Dosage: Isoniazid 5$10 \mathrm{mg} /(\mathrm{kg} \cdot \mathrm{d})$, rifampicin $5-10 \mathrm{mg} /(\mathrm{kg} \cdot \mathrm{d})$, ethambutol $15 \mathrm{mg} /(\mathrm{kg} \cdot \mathrm{d})$, pyrazinamide $20-30 \mathrm{mg} /(\mathrm{kg} \cdot \mathrm{d})$.

\section{Surgical Methods}

After general anesthesia had taken effect, the patient was placed in the prone position and routinely disinfected and toweled. After fluoroscopic positioning on the C-arm, a posterior median incision was made, and the paravertebral muscles were stripped bilaterally to the outer edge of the articular eminence. The appropriate number of pedicle screws were implanted in the normal vertebral body above and below the eroded vertebral body of the TB lesion, and pre-curved connecting rods and nail caps were installed to correct the kyphosis and restore the normal physiological curvature of the vertebrae. Intra-operative fluoroscopy showed that the screw nut was locked in place with good internal fixation. After rinsing with natural saline, the corresponding size of autologous bone particles or allogeneic bone particles were taken, trimmed, and placed in the articular eminence of the diseased vertebral body at the bone-graft bed, and the incision was closed with layer-by-layer sutures. Fifteen pediatric patients underwent second-stage lesion removal using the anterior approach 7 to 14 days after the posterior orthopedic internal fixation had been carried out. The pediatric patient was placed in a supine position with the shoulders elevated so that the cervical spine could be mildly hyperextended to facilitate exposure of the transthoracic junction region. The left sternocleidomastoid muscle was selected as the medial oblique incision approach, and the platysma was incised. In the inner side of the sternocleidomastoid muscle, the space between the cervical vascular sheath and the organ sheath was incised layer by layer to the prevertebral fascia to expose the lesion, and the abscess and caseous necrotic material were removed and sent for pathological examination. The allogeneic bone was then placed in the intervertebral space to support the graft bone.

\section{Postoperative Management}

After surgery, the vital signs were monitored in the pediatric patients, including blood pressure, respiration, and pulse. The drainage tube was removed when the drainage had dropped to $<50 \mathrm{~mL}$ within $24 \mathrm{~h}$ of surgery. A head, neck, and chest brace was worn for 3 days after surgery, and functional floor exercises were performed; the brace needed to be worn for 3-6 months. The implant fusion and internal fixation in patients were evaluated by X-ray, CT, and MRI 1, 3, 6, and 12 months after surgery. During regular quadruple anti-TB treatment, the levels of ESR 


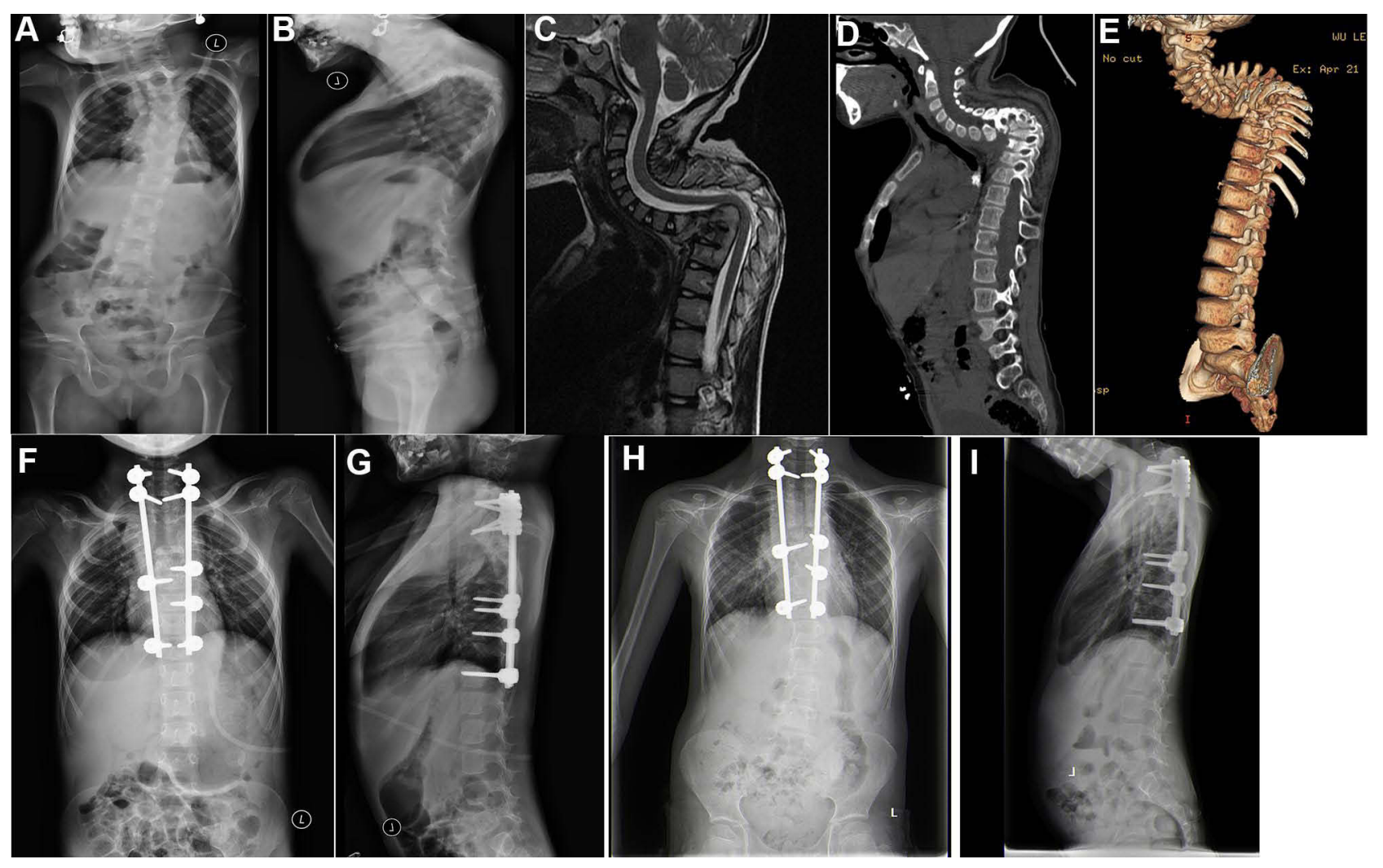

Figure I A female patient of 7 years old with C7-T9 vertebral tuberculosis and kyphosis. One-stage posterior approach internal fixation orthopedics was conducted followed by two-stage anterior approach lesion removal with additional bone grafting. (A and B) The preoperative whole spine frontal and lateral $\mathbf{x}$-ray showed cervicothoracic segmental kyphosis, and obvious C7-T9 localized lateral kyphosis. (C) The preoperative MRI indicated the destruction of the C7 T9 vertebral body and intervertebral disc, a small amount of abscess formation in the paravertebral area, and a change in the intervertebral disc signal. (D and E) The preoperative CT and sagittal $3 \mathrm{D}$ reconstruction suggested multiple bone destruction at C7-T9, with some of the bone edges becoming hyperplastic and sharp. (F and $\mathbf{G})$ The whole spine frontal and lateral $\mathbf{x}$-ray after the staged posterior-anterior combined surgery showed a good position of the internal fixation and a good sequence of cervicothoracic vertebrae. (H and I) The whole spine frontal and lateral $x$-ray one year after surgery showed good internal fixation and complete osseous fusion.

and CRP as well as hepatic and renal function should be checked regularly.

\section{Evaluation of the Therapeutic Effect}

Improvements in the clinical symptoms, orthopedic results, and bone-graft fusion in the pediatric patients were evaluated. The American Spinal Injury Association's (ASIA) spinal-cord injury classification, the Japanese Orthopaedic Association's (JOA) cervical-spine function score, the neck disability index (NDI), and the pain visual analogue score (VAS) were used to assess the functional improvement and quality of life of the patients before and after the operation and at the final follow-up. A whole spine frontal and lateral $\mathrm{X}$-ray was taken in the standing position, and the orthopedic status in the pediatric patients was evaluated by measuring the sagittal and coronal parameters (including the sagittal local Cobb angle, the sagittal vertical axis [SVA], and the coronal balance distance [CBD]). The fusion of the implant was evaluated using the Eck fusion grading criteria. The occurrence of postoperative and follow-up complications, including sinus tract formation, destruction of the bone graft, and vertebral bone destruction, were recorded.

\section{Statistical Analysis}

All statistical analyses were performed using SPSS 22.0 software. The countable data were expressed by frequency or rate, and the measurement data were expressed by mean \pm standard deviation $(\bar{x} \pm \mathrm{s})$. The preoperative, immediately postoperative, and final follow-up imaging measurements and functional scores were analyzed by the $t$-test, and the test level was set at 0.05 bilaterally. $P$ value of $<$ 0.05 was considered statistically significant.

\section{Results}

\section{Surgical Results}

All children successfully completed the operation. The operation duration was $3.56 \pm 0.68 \mathrm{~h}$, with an 

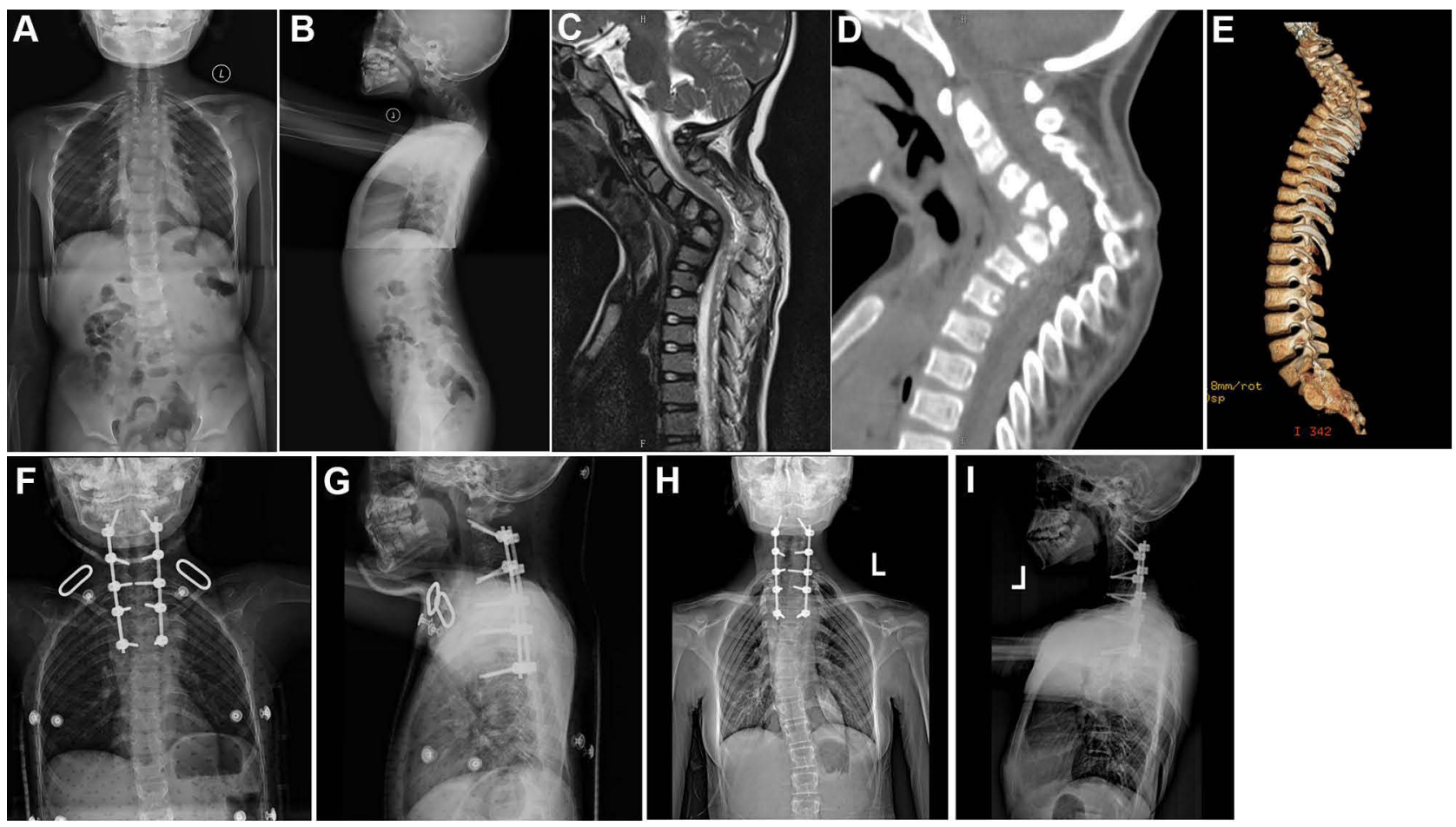

Figure 2 A female patient of 3 years old with C4-T4 vertebral tuberculosis and kyphosis. One-stage posterior approach internal fixation orthopedics was conducted followed by two-stage anterior approach lesion removal with additional bone grafting. (A and B) The preoperative whole spine frontal and lateral $\mathrm{x}$-ray showed obvious $\mathrm{C} 4-$ T4 kyphosis. (C) The preoperative MRI showed destruction of C4 T4 vertebral body and intervertebral disc, with paravertebral abscess formation and change in the intervertebral disc signal. (D and E) The preoperative CT and sagittal 3D reco nstruction suggested C4-T4 multiple arch bone destruction with C6 vertebral body collapse. (F and $\mathbf{G})$ The whole spine frontal and lateral x-ray after the staged posterior-anterior combined surgery showed a good position of the internal fixation. ( $\mathbf{H}$ and $\mathbf{I})$ The whole spine frontal and lateral x-ray six year after surgery showed good internal fixation and complete osseous fusion.

intraoperative hemorrhage of $289.7 \pm 84.3 \mathrm{~mL}$ and an average of fixed segments of $7.3 \pm 1.8$. The average preoperative sagittal local Cobb angle was $67.06 \pm 17.54^{\circ}$, and the average postoperative Cobb angle was $19.48 \pm$ $2.32^{\circ}$. The preoperative SVA was $35.19 \pm 10.69 \mathrm{~mm}$, which improved to $7.67 \pm 1.40 \mathrm{~mm}$ postoperatively. The preoperative CBD was $22.58 \pm 7.59 \mathrm{~mm}$, which improved to $8.99 \pm 1.25 \mathrm{~mm}$ postoperatively. The overall sagittal and coronal balance, together with the local Cobb angle, were significantly improved after surgery (Table 2). The tissues obtained during surgery were pathologically examined, and the results suggested caseous necrosis and lymphocytic infiltration.

\section{Follow-Up Results}

A full follow-up was completed in all the pediatric patients with a follow-up duration of $28.1 \pm 9.7$ months. The sagittal local Cobb angle and parameters concerning the sagittal and coronal balance before and immediately after surgery, together with those at the final follow-up, are shown in Table 2 . There existed statistically significant differences in all the data obtained before and immediately after surgery as

Table 2 Results of Imaging Measurements Before and After Surgery and at the Last Follow-Up in 15 Pediatric Patients

\begin{tabular}{|l|c|c|c|c|c|}
\hline & Preoperative & Postoperative & Final Follow-Up Visit & \multicolumn{2}{|c|}{ Statistical Values } \\
\cline { 3 - 6 } & & & & & $\begin{array}{c}\text { Pre-Op vs Post-Op } \\
\text { Post-Op vs Final } \\
\text { Follow-Up }\end{array}$ \\
\hline The coronal balance distance $(\mathrm{mm})$ & $22.58 \pm 7.59$ & $8.99 \pm 1.25$ & $7.91 \pm 0.61$ & $\mathrm{t}=6.642, \mathrm{P}<0.01$ & $\mathrm{t}=2.635, \mathrm{P}=0.02$ \\
The kyphosis Cobb angle $\left(^{\circ}\right)$ & $67.06 \pm 17.54$ & $19.48 \pm 2.32$ & $18.63 \pm 2.77$ & $\mathrm{t}=10.863, \mathrm{P}<0.01$ & $\mathrm{t}=2.066, \mathrm{P}=0.058$ \\
The sagittal vertical axis(mm) & $35.19 \pm 10.69$ & $7.67 \pm 1.40$ & $6.97 \pm 1.50$ & $\mathrm{t}=10.227, \mathrm{P}<0.01 \quad$ & $\mathrm{t}=4.740, \mathrm{P}<0.01$ \\
\hline
\end{tabular}


Table 3 The Results of JOA Score, VAS, and NDI Before and After Surgery and at the Last Follow-Up in I5 Pediatric Patients

\begin{tabular}{|c|c|c|c|c|c|}
\hline & \multirow[t]{2}{*}{ Preoperative } & \multirow[t]{2}{*}{ Postoperative } & \multirow[t]{2}{*}{ Final Follow-Up Visit } & \multicolumn{2}{|c|}{ Statistical Values } \\
\hline & & & & Pre-Op vs Post-Op & $\begin{array}{c}\text { Pre-Op vs Final } \\
\text { Follow-Up }\end{array}$ \\
\hline JOA Score & $8.93 \pm 3.51$ & $14.67 \pm 1.34$ & $15.80 \pm 0.94$ & $t=7.206, P<0.01$ & $\mathrm{t}=3.371, \mathrm{P}=0.05$ \\
\hline VAS Score & $7.40 \pm 1.35$ & $2.67 \pm 0.62$ & $1.80 \pm 0.56$ & $\mathrm{t}=\mid 4.992, \mathrm{P}<0.01$ & $\mathrm{t}=5.245, \mathrm{P}<0.0 \mathrm{I}$ \\
\hline The neck disability index(NDI) & $32.67 \pm 4.83$ & $13.73 \pm 2.08$ & $9.60 \pm 1.81$ & $t=|6.369, P<0.0|$ & $t=16.163, P<0.01$ \\
\hline
\end{tabular}

well as those acquired at the final follow-up in all the pediatric patients. By the time of the final follow-up, the JOA score had improved from $8.93 \pm 3.51$ before surgery to $14.67 \pm 1.34$, the VAS had improved from $7.40 \pm 1.35$ before surgery to $2.67 \pm 0.62$, and the NDI had improved from $32.67 \pm 4.83$ before surgery to $13.73 \pm 2.08$. Compared with the preoperative period, there were statistical differences in the above indicators postoperatively and at the final followup $(\mathrm{P}<0.05)$ (Table 3$)$. According to the ASIA classification, after surgery, four cases had improved from grade $\mathrm{C}$ preoperatively to grade $\mathrm{E}$, five cases had improved from grade D preoperatively to grade $\mathrm{E}$, three cases had improved from grade B preoperatively to grade E, one case had improved from grade $\mathrm{B}$ preoperatively to grade $\mathrm{D}$, and one case had improved from grade $\mathrm{C}$ preoperatively to grade D. One pediatric patient was evaluated as grade C preoperatively due to severe vertebral destruction and spinal-nerve injury, and no improvement was observed after surgery. Fifteen pediatric patients were evaluated using the Eck fusion grading criteria, and nine patients achieved grade I, five grade II, and one grade III fusion in the implant area, and satisfactory fusion results were obtained at the final follow-up. During the follow-up, one patient had a second surgery $(6.66 \%)$ and two cases had other complications (13.33\%).

\section{Management of Complications}

There were three cases with complications after surgery. One case had a sinus tract after surgery in the area operated on, which was cured after debridement, dressing change, and the adjustment of the anti-TB drugs. Another case had poor recovery of neurological functions after surgery and developed neck and shoulder pain with stiffness, which was significantly improved by wearing a neck brace and using nervenourishing drugs (Vitamin B12) accordingly. The rest of the patients did not suffer from nerve injury of the spinal cord or other important anatomical structures such as the vertebral artery. During the follow-up, one case had a unilateral fracture in the titanium rod, which led to the prolapse of the pedicle screw above the fracture, and a second posterior approach surgery was performed to replace the internal fixation device.

\section{Discussion}

The spine is a common site for extra-pulmonary TB, accounting for about $50 \%$ of osteoarticular $\mathrm{TB},{ }^{14,15}$ although cervicothoracic spinal TB, involving the C7-T3 segments, accounts for only $5 \%$ of all spinal TB. ${ }^{16}$ Due to the special anatomical structure and physiological characteristics of the spine in children, TB lesions are more likely to spread to different segments of the spine or the anterior column of the vertebral body, leading to the destruction and collapse of the vertebral body. ${ }^{17}$ It has been found that the presence of blood vessels in the endplate cartilage in children leads to a greater spread of spinal TB, whether it occurs in the vertebral body or the intervertebral space. ${ }^{18,19}$ Moreover, cervicothoracic TB more often involves the anterior column and easily combines with injury to the adjacent vertebral bodies leading to anterior column instability; however, the posterior and middle columns remain relatively intact. As the vertebral stress load shifts to the anterior column, kyphosis is further exacerbated, which then leads to spinal-cord dysfunction. ${ }^{20}$ Approximately 39\% of pediatric patients with spinal TB develop progressive kyphosis, with $3 \%$ developing a kyphosis angle of more than $60^{\circ} .^{21}$ Although anti-TB drugs are effective in inactivating Mycobacterium TB, the progression of kyphosis cannot be prevented. ${ }^{22}$ Surgical correction of kyphosis is the only effective treatment, and timely surgical treatment can prevent the progression of kyphosis.

For the treatment of cervicothoracic TB with kyphosis in pediatric patients, the surgical option is particularly important. The anterior, posterior, and combined posterior-anterior surgeries are the classic surgical approaches for the treatment of spinal TB. Liang et $\mathrm{al}^{23}$ stated that identifying the risk factors for kyphosis in pediatric patients with spinal TB might be crucial for developing an individualized surgical protocol, and the risk factors were summarized as follows: (1) age $<15$ 
years; (2) severe loss of vertebral body height and poor spinal stability; (3) a kyphosis angle $>30^{\circ}$; (4) kyphosis located at the junction of the cervicothoracic or thoracolumbar segments. In the present study, the average age of the pediatric patients was 6.8 years (3-12 years), and the preoperative sagittal kyphosis angle was $67.06 \pm 17.54^{\circ}$. In all 15 children, there existed destruction of the anterior edge of the vertebral body in the parietal region, with the loss of anterior column height and unbalanced growth of the anterior and posterior columns. Cui et $\mathrm{al}^{24}$ found that there was a high incidence of complications after lesion removal and graft fusion by the simple anterior approach, the most serious of which being secondary kyphosis, and they concluded that graft fusion using this approach weakened the growth of the anterior column of the vertebral body, leading to an imbalance in the growth of the anterior and posterior columns of the vertebral body, thus aggravating the kyphosis. Hu et $\mathrm{al}^{25}$ concluded that one-stage posterior lesion removal with fusion and internal fixation achieved good results in the treatment of spinal TB with kyphosis. The advantages of this surgical approach were as follows: (1) it was less traumatic; (2) the removal of the TB lesion could be completed at the same time as the internal fixation of the bone graft, thus avoiding secondary surgery; (3) complex anatomy could be avoided during the anterior approach; (4) abscesses and lesions were removed from the vertebral or peri-vertebral body with a $270^{\circ}$ field of view. In the present study, we concluded that this surgical approach was limited by the inability to completely remove the TB lesion when the abscess was present in the anterior column of the vertebral body or when the paravertebral abscess covered a large area and involved multiple vertebral bodies.

In the present study, all pediatric patients had combined neurological injury, and when an abscess formed in the anterior part of the vertebral body and involved multiple segments, anterior surgery was able to directly expose the anterior structures of the vertebral body, remove the lesion under direct vision, obtain complete spinal-cord decompression, and create conditions for anterior support bone grafting. With correction by surgery using the posterior approach, good surgical maneuvering space was available in the second-stage anterior supplemental bone graft. In a pediatric patient with severe kyphosis (Figure 1), there existed severe bone destruction and vertebral collapse; thus, the spinal cord was often stretched and compressed, and the ischemia of the spinal cord might cause a decrease in tolerance to surgery. Therefore, in the pediatric patient with severe kyphosis, it was important to first ensure effective decompression around the spinal cord and to choose the most appropriate orthopedic technique. Posterior long segment fixation fusion was adopted in the present study because it was not possible to achieve stable reconstruction by simply fusing the upper and lower two segments of the diseased spine without the increased risk of the postoperative internal fixation becoming loose and breakage. As reported in the previous literature, the common surgical approach for the treatment of spinal TB with kyphosis in the pediatric patient is still the combined posterior-anterior surgery, which has the advantages of preventing the occurrence of curvature, ensuring the uniform developmental balance of the anterior and posterior columns of the vertebrae, and avoiding the aggravation of kyphosis in the later stages. ${ }^{26}$ It has been reported that combined posterior-anterior surgery can be accomplished in one stage. ${ }^{9,27}$ However, in the present study, the average age of the pediatric patients was 6.8 years, and the TB lesions were all present in the cervicothoracic segment, which made the onestage posterior-anterior combined surgery treatment risky and poorly tolerated by the patients. The staged posterior-anterior combined surgery for cervicothoracic TB with kyphosis in pediatric patients might have a shorter single-operation duration, it could increase the safety of the operation and completely remove the lesions, sufficiently decompress the spinal nerves, result in firm fixation, and effectively correct the kyphosis.

In surgery for cervicothoracic TB with kyphosis, the radical scraping of endplate cartilage should be avoided with the premise of complete clearance of the TB lesion, which would aggravate the instability of the anterior column support and disrupt the normal remodeling ability of the spine, leading to aggravation of the kyphosis during future growth and development. $^{28,29}$ In the 15 pediatric patients in the present study, a portion of the growth plate was preserved as much as possible during the anterior surgical abscess removal, and a supporting bone graft was provided to create more secure fixation and correct the kyphosis. In the present study, pediatric patient deformities achieved good orthopedic results postoperatively. The posterior kyphosis Cobb angle in the 15 patients was $67.06 \pm 17.54^{\circ}$ before surgery and was corrected to $19.48 \pm 2.32^{\circ}$ after surgery, with a correction rate of $86.6 \%$. The Cobb angle was $18.63 \pm 2.77^{\circ}$ at the final follow-up, and there was no statistically significant difference between the follow-up results and the postoperative measurements $(\mathrm{t}=$ $0.368, \mathrm{P}>0.05$ ). Therefore, considering the aims of the treatment for spinal TB in pediatric patients, it is important to consider not only the complete removal of the lesion and the restoration of spinal-nerve dysfunction but also the potential for maintaining balanced spinal growth and preserving spinal remodeling capacity, which is particularly important for the 
correction of spinal stability and sagittal and coronal imbalances.

From the long-term follow-up results of the staged posterior-anterior combined surgery for cervicothoracic segment kyphosis in pediatric patients, we believe that staged posterior-anterior combination surgery might be an option for pediatric patients under the following conditions: (1) less tolerance or inability to tolerate the one-stage surgery; (2) a severe collapse of the vertebral body, with arch-bone destruction and severe sagittal and coronal imbalance; (3) severe small-joint separation and laterally shifted spinal instability in the segment with kyphosis; (4) multi-segmental ( $\geq 3$ segments) cervicothoracic TB; (5) the destruction of both anterior and posterior columns with obvious anterior abscesses; (6) an unstable internal fixation via the anterior approach or an insecure implant and insufficient support via the anterior approach alone.

The present study had some limitations. Because it was a single-center retrospective study, the number of cases selected was relatively small and the follow-up period was not long enough. The effects of implant fusion on the distal vertebral body and degenerative changes and the future spinal growth and development in pediatric patients after surgery still deserve further study. Considering the young age of the patients, the selected assessment indicators might be subject to error. Therefore, the conclusion in the present study still needs to be confirmed by a large sample of controlled trials in multiple medical centers.

\section{Conclusion}

In the present study, satisfactory results were achieved in the treatment of pediatric patients with cervicothoracic TB and kyphosis by staged posterior-anterior combined surgery. The kyphosis was significantly corrected, neurological decompression was achieved safely and effectively, and good spinal neurological recovery and bone graft fusion had been achieved by the time of the medium and long-term follow-up.

\section{Ethical Statement}

This study was conducted with approval from the Ethics Committee of Xinjiang Medical University Affiliated First Hospital. This study was conducted in accordance with the declaration of Helsinki. Written informed consent was obtained from guardians of all participants under 16 years old.

\section{Funding}

Project Title: Research on TGF- $\beta 2$ in regulating the apoptotic signaling pathways of Fluoride-stained Cells Project (No: 2018D01C158).

\section{Disclosure}

The authors report no conflicts of interest in this work.

\section{References}

1. Rajasekaran S. Kyphotic deformity in spinal tuberculosis and its management. Int Orthop. 2012;36(2):359-365. doi:10.1007/s00264011-1469-2

2. Jain AK, Sreenivasan R, Mukunth R, Dhammi IK. Tubercular spondylitis in children. Indian $J$ Orthop. 2014;48(2):136-144. doi:10.4103/0019-5413.128747

3. Liang W, Zhang J, Ren Z, et al. Comparison of the clinical efficacy of 3 surgical methods for treating spinal tuberculosis in children. Med Sci Monit. 2020;26:e926142. doi:10.12659/MSM.926142

4. Jain AK, Kumar J. Tuberculosis of spine: neurological deficit. Eur Spine J. 2013;22(Supp14):624-633. doi:10.1007/s00586-012-2335-7

5. Subramani S, Shetty AP, Kanna RM, Rajasekaran S. A rare cause of late onset neurological deficit in post tuberculous kyphotic deformity-case report. $J$ Spine Surg. 2017;3(4):740-743. doi:10.21037/jss.2017.12.01

6. Rajasekaran S, Prasad shetty A, Dheenadhayalan J, Shashidhar Reddy J, Naresh-Babu J, Kishen T. Morphological changes during growth in healed childhood spinal tuberculosis: a 15-year prospective study of 61 children treated with ambulatory chemotherapy. J Pediatr Orthop. 2006;26(6):716-724. doi:10.1097/01.bpo.0000230 326.21707 .71

7. Issack PS, Boachie-Adjei O. Surgical correction of kyphotic deformity in spinal tuberculosis. Int Orthop. 2012;36(2):353-357. doi:10.1007/s00264-011-1292-9

8. Jain AK, Dhammi IK, Jain S, Mishra P. Kyphosis in spinal tuberculosis - prevention and correction. Indian J Orthop. 2010;44 (2):127-136. doi:10.4103/0019-5413.61893

9. Zhu Z, Hao D, Wang B, et al. Selection of surgical treatment approaches for cervicothoracic spinal tuberculosis: a 10 -year case review. PLoS One. 2018;13(2):e0192581. doi:10.1371/journal. pone.0192581

10. Abulizi Y, Liang WD, Maimaiti M, Sheng WB. Smith-Petersen osteotomy combined with anterior debridement and allografting for active thoracic and lumbar spinal tuberculosis with kyphotic deformity in young children: a prospective study and literature review. Medicine. 2017;96(32):e7614. doi:10.1097/MD.0000000000007614

11. Zhang $\mathrm{HQ}$, $\mathrm{Hu} \mathrm{X}$, Yin $\mathrm{X}$, Chen $\mathrm{Y}$. One-stage combined anterior-posterior approach treatment of multiple cervicothoracic spinal tuberculosis with kyphosis. Int Orthop. 2015;39 (8):1605-1610. doi:10.1007/s00264-015-2778-7

12. Yin $\mathrm{H}$, Wang K, Gao Y, et al. Surgical approach and management outcomes for junction tuberculous spondylitis: a retrospective study of 77 patients. J Orthop Surg Res. 2018;13(1):312. doi:10.1186/ s13018-018-1021-9

13. Zeng H, Shen X, Luo C, et al. Comparison of three surgical approaches for cervicothoracic spinal tuberculosis: a retrospective case-control study. J Orthop Surg Res. 2015;10:100. doi:10.1186/ s13018-015-0238-0

14. Wang YX, Zhang HQ, Li M, et al. Debridement, interbody graft using titanium mesh cages, posterior instrumentation and fusion in the surgical treatment of multilevel noncontiguous spinal tuberculosis in elderly patients via a posterior-only. Injury. 2017;48(2):378-383. doi:10.1016/j.injury.2016.12.025 
15. Li ZW, Li ZQ, Tang BM, et al. Efficacy of one-stage posterior debridement and bone grafting with internal fixation in the treatment of monosegmental thoracolumbar tuberculosis. World Neurosurg. 2019;121:e843-e851. doi:10.1016/j.wneu.2018.09.234

16. Hsu LC, Leong JC. Tuberculosis of the lower cervical spine (C2 to C7). A report on 40 cases. J Bone Joint Surg Br. 1984;66(1):1-5. doi:10.1302/0301-620X.66B1.6693464

17. Rajasekaran S, Khandelwal G. Drug therapy in spinal tuberculosis. Eur Spine J. 2013;22(Suppl 4):587-593. doi:10.1007/s00586-0122337-5

18. Komiyama M. [Functional vascular anatomy of the spine and spinal cord]. No Shinkei Geka. 2013;41(6):481-492. Japanese.

19. Yin X, Liu P, Liu YY, Fan WL, Liu BY, Zhao JH. Utilization of ring-shaped bone allograft for surgical treatment of adolescent post-tubercular kyphosis: a retrospective study. Medicine. 2017;96 (24):e7132. doi:10.1097/MD.0000000000007132

20. Zeng H, Shen X, Luo C, et al. 360-degree cervical spinal arthrodesis for treatment of pediatric cervical spinal tuberculosis with kyphosis. BMC Musculoskelet Disord. 2016;17:175. doi:10.1186/s12891-0161034-7

21. Zhang HQ, Lin MZ, Guo HB, Ge L, Wu JH, Liu JY. One-stage surgical management for tuberculosis of the upper cervical spine by posterior debridement, short-segment fusion, and posterior instrumentation in children. Eur Spine J. 2013;22(1):72-78. doi:10.1007/ s00586-012-2544-0

22. Zhang H, Guo Q, Wang Y, Guo C, Tang M. The efficiency of the posterior-only approach using shaped titanium mesh cage for the surgical treatment of spine tuberculosis in children: a preliminary study. J Orthop Surg. 2018;26(3):2309499018806684. doi:10.1177/ 2309499018806684
23. Liang Q, Wang Q, Long G, et al. Clinical effectiveness of the posterior affected-vertebrae fixation method in posterior-anterior surgery to treat thoracic spinal tuberculosis. World Neurosurg. 2019;123:29-39. doi:10.1016/j.wneu.2018.11.199

24. Cui X, Li LT, Ma YZ. Anterior and posterior instrumentation with different debridement and grafting procedures for multi-level contiguous thoracic spinal tuberculosis. Orthop Surg. 2016;8(4):454-461. doi:10.1111/os. 12288

25. Hu X, Zhang H, Yin X, Chen Y, Yu H, Zhou Z. One-stage posterior focus debridement, fusion, and instrumentation in the surgical treatment of lumbar spinal tuberculosis with kyphosis in children. Childs Nerv Syst. 2016;32(3):535-539. doi:10.1007/s00381-015-2948-3

26. Yin XH, Zhou ZH, Yu HG, Hu XK, Guo Q, Zhang HQ. Comparison between the antero-posterior and posterior only approaches for treating thoracolumbar tuberculosis (T10-L2) with kyphosis in children: a minimum 3-year follow-up. Childs Nerv Syst. 2016;32(1):127-133. doi:10.1007/s00381-015-2935-8

27. Zhang HQ, Wang YX, Guo CF, et al. One-stage posterior approach and combined interbody and posterior fusion for thoracolumbar spinal tuberculosis with kyphosis in children. Orthopedics. 2010;33 (11):808. doi:10.3928/01477447-20100924-10

28. Schulitz KP, Kothe R, Leong JC, Wehling P. Growth changes of solidly fused kyphotic bloc after surgery for tuberculosis. Comparison of four procedures. Spine. 1997;22(10):1150-1155. doi:10.1097/00007632-199705150-00016

29. Moon MS, Kim SS, Lee BJ, Moon JL. Spinal tuberculosis in children: retrospective analysis of 124 patients. Indian J Orthop. 2012;46 (2):150-158. doi:10.4103/0019-5413.93676
International Journal of General Medicine

\section{Publish your work in this journal}

The International Journal of General Medicine is an international, peer-reviewed open-access journal that focuses on general and internal medicine, pathogenesis, epidemiology, diagnosis, monitoring and treatment protocols. The journal is characterized by the rapid reporting of reviews, original research and clinical studies across all disease areas. The manuscript management system is completely online and includes a very quick and fair peer-review system, which is all easy to use. Visit http://www.dovepress.com/ testimonials.php to read real quotes from published authors. 